\title{
Blood serum DSC analysis of well-trained men response to CrossFit training and green tea extract supplementation
}

\author{
Anna Michnik ${ }^{1,2}$ (1) Ewa Sadowska-Krępa ${ }^{3} \cdot$ Przemysław Domaszewski $^{4} \cdot$ \\ Klaudia Duch $^{1,2} \cdot$ Ilona Pokora ${ }^{3}$
}

Received: 18 January 2017/Accepted: 20 March 2017/Published online: 4 April 2017

(c) The Author(s) 2017. This article is an open access publication

\begin{abstract}
Differential scanning calorimetry (DSC) has been used for the detection of post-exercise changes in blood serum resulting from participation in the CrossFit (CF) training combined with green tea extract (GTE) supplementation. Blood samples from 20 well-trained men were collected at rest, immediately post-exercise and after $1 \mathrm{~h}$ of recovery in two trials: first before and second after CF training combined with GTE or placebo administration in the supplemented (S) and control (C) groups, respectively. Selected muscle damage biomarkers have been compared in different phases of the experiment. A significant increase in blood lactate content has been observed post-exercise in both trials in both participants' groups. The opposite trends have been noted for the $\mathrm{C}$ and $\mathrm{S}$ groups in creatine kinase $(\mathrm{CK})$ activity changes recorded during the first to the second trial: an increase in CK for the control and a decrease for the supplemented group in all phases of the experiment: pre-exercise, post-exercise and after recovery. In the second trial, all CK values for the S group have been found significantly lower than the corresponding values recorded in the $\mathrm{C}$ group. These results suggest a
\end{abstract}

Anna Michnik

anna.michnik@us.edu.pl

1 Department of Medical Physics, A. Chełkowski Institute of Physics, University of Silesia, ul. Uniwersytecka 4, 40-007 Katowice, Poland

2 The Silesian Centre for Education and Interdisciplinary Research, ul. 75 Pułku Piechoty 1A, 41-500 Chorzów, Poland

3 Department of Physiological and Medical Sciences, The Jerzy Kukuczka Academy of Physical Education in Katowice, ul. Mikołowska 72a, 40-065 Katowice, Poland

4 Division of Tourism and Recreation, Faculty of Physical Education and Physiotherapy, Opole University of Technology, Opole, Poland mitigate effect of GTE supplementation on post-training muscle damage. DSC results did not reveal clear effects of training nor GTE supplementation on serum denaturation transition. However, interesting dependences of thermodynamic parameters describing this transition have been observed in different phases of the experiment. Statistically significant negative correlations have been found between post-training $\mathrm{VO}_{2 \max }$ and post-exercise thermodynamic parameters associated with haptoglobin contribution to serum denaturation transition.

Keywords CrossFit training - DSC - Exercise · Green tea extract $\cdot$ Human blood serum

\section{Introduction}

The consumption of exogenous supplemental antioxidants among athletes has increased despite the lack of clear evidence of their benefits. The majority of published papers confirm the general belief that antioxidant supplements improve health and even prevent several diseases. However, there is also a growing evidence regarding the useless or negative effects of antioxidant supplementation on physiological and biochemical outcomes and performance of exercise-trained individuals [1-4].

After many years of intensive research, it is now well documented that exhaustive exercise induces production of reactive oxygen species (ROS) resulting in increased oxidative stress, which may lead to the damage of cellular components, including proteins, lipids, carbohydrates and nucleic acids. So, a great body of researchers have investigated the possibility to prevent the exercise-induced oxidative stress and muscle damage through nutritional 
intervention, mainly using antioxidants. For a comprehensive review and discussion on this topic, please refer, e.g., to [4-6]. On the other hand, recent investigations report also several positive aspects of ROS generation in sport performance and their role in cell signaling and adaptation to regular training [7-9]. ROS play an important role in lipid peroxidation which leads to an increase in permeability of cell skeletal membranes, followed by a release of cytosolic proteins and enzymes such as creatine kinase (CK) and lactate dehydrogenase (LDH) into the blood [10]. Moreover, if oxidative stress levels are already low, which may be the case in healthy young individuals and athletes, the exogenous antioxidant supplementation may be detrimental. Thus, the relationship between exercise, oxidative stress and supplementation remains a complex issue.

Tea is a rich natural source of flavonoid antioxidants from the polyphenol family. Particularly, green tea extracts with antioxidant, anti-carcinogen, anti-inflammatory and anti-radiation properties have been a focus of recent scientific research. Beneficial effects of green tea or its extracts against cancer, obesity, metabolic syndrome and cardiovascular disease have been reported [11-13]. Antioxidant properties of green tea are associated with the presence of several polyphenols, primarily catechins including epigallocatechin gallate (EGCG), (-) epicatechin gallate (ECG), (-) epicatechin (EC) and (-) epigallocatechin (EGC) [14]. Zhang et al. [15] have recently shown that long-term supplementation with green tea polyphenol could provide an efficient protection against the health issues induced by high-voltage power lines. They found that 12 months of green tea polyphenol supplementation (GTPS) significantly diminished the negative effects of extremely low-frequency electromagnetic fields (ELF-EMFs) on the health of the exposed workers. However, 3 months after GTPS withdrawal, no protective effects of previous supplementation on oxidative stress and oxidative damage to DNA were observed.

Studies examining the effects of green tea consumption on exercise-induced oxidative stress and exercise metabolism in humans are rather scarce and results of these studies are often inconclusive [16, 20]. Previous studies have generally found that antioxidant supplements do not improve performance; however, there are many reports that exogenous antioxidant supplements may strengthen antioxidant defense system in healthy exercising humans and limit or even prevent the exercise-induced tissue damage or help the sportsmen to recover from the damage [21-23]. Richards et al. [17] have reported an increase in sport performance after green tea catechins (GTC) ingestion, namely a short-term ingestion of EGCG caused $\mathrm{VO}_{2-}$ max increase in 14 out of 19 subjects. Jówko et al. [18] have reported that a long-term (4-week) green tea extract (GTE) supplementation enhanced plasma total polyphenols at rest as well as 5 min after the muscular endurance test and it contributed to the rise of resting total antioxidant status in plasma of previously untrained men. They concluded that dietary supplementation with GTE may provide protection against oxidative damage induced by both short-term muscular endurance test and long-term strength training. In another Jówko et al.'s study [19], green tea catechins-treated soccer players were able to perform a higher number of lift repetitions during the muscle endurance test, but none of the analyzed plasma biomarkers was affected by the ingestion of green tea catechins, suggesting that a $640 \mathrm{mg}$ dose was too low to attenuate exercise-induced oxidative stress and muscle damage. Hodgson et al. [24] found that in healthy physically active men, GTE enhanced lipolysis and fat oxidation when compared to placebo, but only under resting conditions, whereas no effect of GTE was seen during exercise. In a more recent study, Sugita et al. [20] have investigated the effects of green tea catechins on oxidative stress metabolites in healthy individuals both at rest and during exercise. The overall data presented in this study suggested that GTC (a single dose of $780 \mathrm{mg}$ ) could improve physical performance particularly in terms of endurance capacity expressed as $\mathrm{VO}_{2 \max }$ in a physically active general population.

Differential scanning calorimetry (DSC) has recently been found useful in the support of various medical diagnoses [25-36] by analysis of plasma or serum heat capacity changes in the temperature range of protein components thermal denaturation. DSC profiles of biofluids are able to reflect their modified composition, changes in thermal stability of major proteome components resulting from covalent modifications or binding interactions involving disease biomarkers. Generally, DSC profile of serum/plasma heat capacity changes provides information on the health status of blood donor based on the individual thermal characteristics. Thus, it seems justified to use the calorimetric analyses also for the evaluation of the beneficial or detrimental effects of physical activity or supplementation. Such innovative attempts of DSC adoption for the resolving of some problems in sport medicine were made by us recently $[37,38]$.

The purpose of the current study was to evaluate the effects of green tea extract supplementation in combination with CrossFit training lasting for 6 weeks on the physical performance and recovery after a short-term exercise in well-trained men. In addition to the typical evaluation of blood biomarkers of muscle damage, such as the activity of lactate dehydrogenase (LDH) and creatine kinase (CK), nonconventional approach has been applied. Using DSC method, blood serum samples taken from the participants during two trials: before and after a 6-week CrossFit training before, immediately after and $1 \mathrm{~h}$ after the exercise test have been analyzed. 


\section{Experimental}

\section{Participants of the experiment}

Twenty nonsmoking male students of the Opole University of Technology, volunteers involved in CrossFit training (CF training), were randomized between the following groups: the control group-receiving placebo (C) and the group supplemented with the green tea extract $(\mathrm{S})$. All subjects were informed about the purpose and the nature of the research before giving their written consent to participate in the experiment, which had been approved by the Ethics Committee of the Jerzy Kukuczka Academy of Physical Education in Katowice (No. 4/2013). The exclusion criteria were the use of tobacco products, alcohol consumption, the use of any medicine or dietary supplements during 4 weeks prior to the study.

\section{Experiment design}

All supplements were administrated in soft gelatinous capsules (Olimp Labs, Dębica, Poland) at a dose of two capsules once a day for 6 weeks. One placebo capsule contained microcrystalline cellulose, magnesium stearate and maltodextrin instead of plant extract. One GTE capsule contained $250 \mathrm{mg}$ of standardized GTE (245 polyphenols including $200 \mathrm{mg}$ catechins among $137 \mathrm{mg}$ epigallocatechin-3-gallate) and additional substances such as maltodextrin, microcrystalline cellulose and magnesium stearate. The daily doses of GTE extract were treated according to the manufacturer's instructions. In case of adverse events associated with dietary supplements, all subjects could opt out of taking part in this study.

All students participated an incremental maximal oxygen uptake $\left(\mathrm{VO}_{2 \mathrm{max}}\right)$ test on cycle ergometer (Sport Excalibur) at two occasions, i.e., before the CF training and supplementation (first trial) and then once more after 6 weeks of $\mathrm{CF}$ training and supplementation (second trial). After a short warm-up cycling, the subjects cycled in 3-min phases starting at a power output of $40 \mathrm{~W}$, and increasing progressively by $40 \mathrm{~W}$. The exercise was continued until exhaustion.

\section{Blood serum samples}

Blood samples were drawn from antecubital vein either into test tubes anticoagulated with heparin or containing clot activator for collection, respectively, of plasma or serum samples at rest, $3 \mathrm{~min}$ after exercise and after $1 \mathrm{~h}$ of passive recovery. Fresh plasma samples were assayed for activities of creatine kinase (CK), lactate dehydrogenase (LDH) and concentrations of lactate (LA) using diagnostic kits from Randox Laboratories (CK522, LD3818 and LC2389, respectively).
Serum samples were stored at $-20{ }^{\circ} \mathrm{C}$ before analysis. For DSC experiment, serum samples were diluted 20 -fold with distilled degassed water. The $\mathrm{pH}$ value of the diluted samples has been within the range 6.5-7.0.

\section{DSC experiment}

DSC measurements were taken on the VP DSC MicroCal instrument (Northampton, MA) in the temperature range $20-100{ }^{\circ} \mathrm{C}$ with the heating rate $1{ }^{\circ} \mathrm{C} \mathrm{min}^{-1}$. A constant pressure of about $1.8 \mathrm{~atm}$ was exerted on the liquids in the cells. The calorimetric data were corrected for the instrumental baseline water-water. DSC curves were normalized for the gram mass of protein and next a linear baseline was subtracted. An apparent excess specific heat capacity $\mathrm{Cp}^{\mathrm{ex}}$ $\left(\mathrm{J}^{\circ} \mathrm{C}^{-1} \mathrm{~g}^{-1}\right)$ versus temperature $\left({ }^{\circ} \mathrm{C}\right)$ has been plotted.

The following parameters of observed DSC transitions have been determined: temperatures of local peak maxima $T_{\mathrm{m}}(m=1,2,3)$, excess specific heat capacities at these temperatures $\mathrm{Cp}_{\mathrm{m}}$, the enthalpy $(\Delta H)$ of serum denaturation (calculated as the area under the endothermic peak, expressed in $\mathrm{J} \mathrm{g}^{-1}$ ) and the width of peak in its half height (HHW). All other experimental conditions and DSC data analysis were practically the same as during the earlier DSC study of human blood serum and described in [37].

\section{Statistical analysis}

Statistical analysis was performed using the Statistica 12 software. For all measures, descriptive statistics were calculated. Analysis of variance (ANOVA) with the stage of experiment as a repeated measure and the group as categorical variable was used. Mauchly's test for sphericity was included as a part of the procedure. If repeated measures ANOVA was statistically significant, Tukey's post hoc test was applied. Student's t test was used to compare the mean values between the two independent groups. The accepted level of significance was taken as $p<0.05$.

When the results of Shapiro-Wilk test revealed that the distributions of the studied variables differed from normal data or in the case of nonhomogenous variances of analyzed groups (in Leven's test $p<0.05$ ), appropriate nonparametric tests were used for comparative analyses.

Pearson's correlation coefficients were found to describe the relationships between biochemical and thermodynamic blood serum parameters.

\section{Results and discussion}

In Table 1, basic characteristics of two groups of participants of the study are given. No significant differences were found between the $\mathrm{C}$ and $\mathrm{S}$ groups in regard to age, 
Table 1 Characteristics of participants

\begin{tabular}{|c|c|c|}
\hline & $\mathrm{C}$ group $(n=9)$ & $\mathrm{S}$ group $(n=11)$ \\
\hline Age/years & $24.1 \pm 2.7$ & $22.5 \pm 2.6$ \\
\hline Height $/ \mathrm{cm}$ & $179.7 \pm 7.8$ & $181.1 \pm 5.3$ \\
\hline Body mass before $\mathrm{CF}$ training $/ \mathrm{kg}$ & $79.7 \pm 10.8$ & $79.9 \pm 7.0$ \\
\hline Body mass after $\mathrm{CF}$ training $/ \mathrm{kg}$ & $79.4 \pm 10.6$ & $80.0 \pm 7.0$ \\
\hline $\mathrm{VO}_{2 \max }$ before $\mathrm{CF}$ training $/ \mathrm{mL} \min ^{-1} \mathrm{~kg}^{-1}$ & $45.7 \pm 5.0$ & $46.5 \pm 5.2$ \\
\hline $\mathrm{VO}_{2 \max }$ after $\mathrm{CF}$ training $/ \mathrm{mL} \min ^{-1} \mathrm{~kg}^{-1}$ & $47.6 \pm 4.1$ & $48.2 \pm 4.3$ \\
\hline $\mathrm{d} \mathrm{VO}_{2 \max } * / \mathrm{mL} \min ^{-1} \mathrm{~kg}^{-1}$ & $1.9 \pm 2.7$ & $1.7 \pm 4.6$ \\
\hline
\end{tabular}

Values are means $\pm \mathrm{sd}$

* The mean value of differences between $\mathrm{VO}_{2 \max }$ after and before $\mathrm{CF}$ training

height and body mass, as well as in $\mathrm{VO}_{2 \max }$ values before the training. The placebo group has demonstrated something greater improvements in respiratory function with training $\left(\mathrm{dVO}_{2 \max }=1.9 \pm 2.7\right)$ compared with GTE-supplemented group $\left(\mathrm{dVO}_{2 \max }=1.7 \pm 4.6\right)$, but the difference was within the experimental error.

Blood biomarkers presented in Table 2 indicate that before the $\mathrm{CF}$ training, the mean values of LA concentration and CK activity were similar in both groups, while the level of LDH activity was significantly higher $(p=0.01$ in Student's $t$ test) in the group assigned to supplementation. It is probably a random difference and its reason is unknown especially that both the resting and post-exercise activities of $\mathrm{LDH}$ were within the references ranges, i.e., 230-460 $\mathrm{U} \mathrm{L}^{-1}$. After the training LDH activity remained unchanged in GTE-supplemented group and slightly increased in placebo group. LA level significantly increased after the exercise in both trials for both groups. The highest CK activity occurred for the $\mathrm{C}$ group after the exercise performed in the second trial. Differences between this $\mathrm{CK}$ value and all values (pre-exercise, post-exercise and after the recovery) in the first trial for the $\mathrm{C}$ group have been found statistically significant. Generally, a slightly higher post-exercise $\mathrm{CK}$ values were observed compared to other phases of the experiment (see Table 2). However, big standard deviations due to high individual variability diminish the perceived tendency. It seems important that the opposite trends have appeared for the placebo and GTE-supplemented groups in all CK activity changes from the first to the second trial: an increase for the first and a decrease for the second of said groups. Statistical analysis has revealed significant differences between $\mathrm{C}$ and $\mathrm{S}$ groups in post-training $\mathrm{CK}$ activities for all stages of experiment $(p=0.03, p=0.008$ and $p=0.02$ for preexercise, post-exercise and after the recovery differences, respectively). For GTE-supplemented group, post-exercise $\mathrm{CK}$ activity before the training has been found significantly higher than post-training pre-exercise and after $1 \mathrm{~h}$ of rest values. These results suggest that supplementation of tea polyphenols, at doses recommended according to manufacturer's instructions, may diminish post-training skeletal muscle damage through suppressive action on oxidative stress and inflammation [21].

Averaged serum DSC curves for $\mathrm{C}$ and $\mathrm{S}$ groups of men are shown in Fig. 1. Both curves are very similar and differences between them are within the experimental error, besides the temperature range above $80{ }^{\circ} \mathrm{C}$. The complex endothermic transition observed for serum solution represents the denaturation of serum proteins, which proceeds over the approximate temperature range $40-90{ }^{\circ} \mathrm{C}$. Most commonly three local maxima can be noticed at $T_{1}, T_{2}$ and $T_{3}$. However, sometimes, $T_{1}$ or $T_{2}$ is absent. For this reason, the first maximum and the second maximum are not well visible in the averaged curves presented in Fig. 1. An contribution connected with protein aggregation is noticeable, particularly in the higher temperature range. The thermal profiles of heat capacity changes for aqueous solutions of human blood serum from athletes have been described and discussed in details earlier [37]. Comparing DSC curves of athletes serum diluted with distilled water (pH 6.5-7.0) with curves reported for serum/plasma of healthy persons where the final $\mathrm{pH}$ of solutions was in the range 7.2-7.5 [25-27, 29-36], distinct differences are visible. The main origin of these differences is probably the thermal denaturation profile of fatty acid-free fraction of albumin. DSC transition representing unfolding of this protein was shown much sharper in buffer $(\mathrm{pH}$ 7.2) than in water (pH 6.5) solution (see Fig. 1a in [39]).

The averaged effects of $\mathrm{CF}$ training in the $\mathrm{C}$ group and training plus supplementation in the $\mathrm{S}$ group on DSC serum profiles in pre-exercise stages are presented in Fig. 2a, b, respectively. In both cases, the differences between DSC curves corresponding to serum before and after the training are very small, and the bigger one seems to be that for the 
Table 2 Changes in LA concentration, $\mathrm{CK}$ and LDH activities induced by the CF training with concomitant supplementation with placebo $(n=9)$ or GTE $(n=11)$

\begin{tabular}{|c|c|c|c|c|c|}
\hline & & \multicolumn{2}{|l|}{$\mathrm{C}$ group } & \multicolumn{2}{|l|}{$\mathrm{S}$ group } \\
\hline & & First trial & Second trial & First trial & Second trial \\
\hline \multirow[t]{2}{*}{$\mathrm{LA} / \mathrm{mmol} \mathrm{L}^{-1}$} & be & $1.38 \pm 0.52$ & $1.13 \pm 0.46$ & $1.47 \pm 0.52$ & $1.30 \pm 0.50$ \\
\hline & ae & $10.72 \pm 2.87$ & $10.07 \pm 2.92$ & $9.06 \pm 1.63$ & $9.40 \pm 2.57$ \\
\hline \multirow[t]{3}{*}{$\mathrm{CK} / \mathrm{U} \mathrm{L}^{-1}$} & be & $214 \pm 49$ & $260 \pm 88$ & $206 \pm 48$ & $188 \pm 43$ \\
\hline & ae & $233 \pm 54$ & $286 \pm 89$ & $242 \pm 46$ & $199 \pm 37$ \\
\hline & $\mathrm{r} 1 \mathrm{~h}$ & $218 \pm 46$ & $241 \pm 54$ & $214 \pm 41$ & $190 \pm 39$ \\
\hline \multirow[t]{3}{*}{$\mathrm{LDH} / \mathrm{U} \mathrm{L}^{-1}$} & be & $242 \pm 42$ & $291 \pm 37$ & $306 \pm 54$ & $305 \pm 29$ \\
\hline & ae & $291 \pm 40$ & $309 \pm 50$ & $362 \pm 61$ & $335 \pm 18$ \\
\hline & $\mathrm{r} 1 \mathrm{~h}$ & $262 \pm 41$ & $295 \pm 44$ & $311 \pm 46$ & $312 \pm 36$ \\
\hline
\end{tabular}

Values are means \pm s.d., be before the exercise, ae $3 \mathrm{~min}$ after the exercise, $r l \mathrm{~h}$ after $1 \mathrm{~h}$ of passive recovery

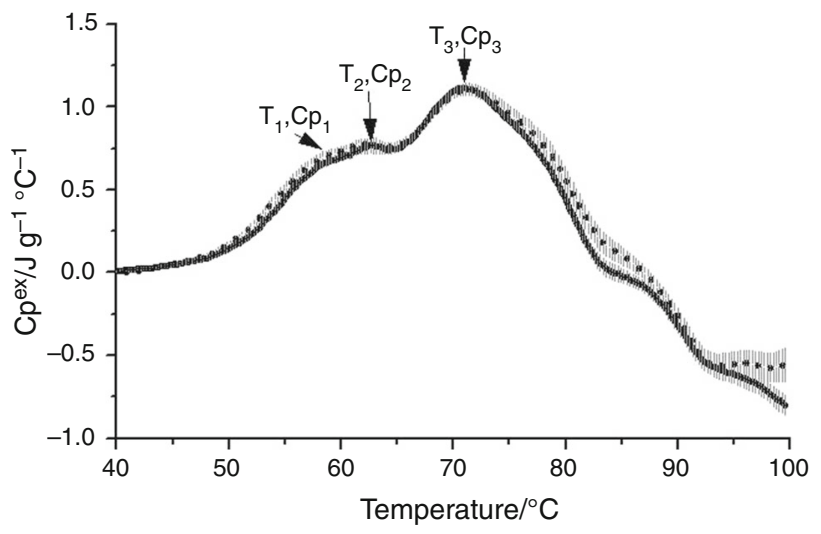

Fig. 1 Mean serum DSC curves for participants from C (solid lines) $(n=9)$ and $\mathrm{S}$ (dotted lines) $(n=11)$ groups; the shadow represents standard errors

control placebo group C. These small averaged posttraining changes appear in the region of $T_{1}$ for the $\mathrm{C}$ group and around $T_{2}$ for the $\mathrm{S}$ group.

Although mean DSC curves have been practically unchanged through the CF training and GTE supplementation, in individual cases, significant differences in heat capacity thermal profile of participants serum could be observed. Examples of such meaningful differences are shown in Fig. 3a, b for selected participants from $\mathrm{C}$ and $\mathrm{S}$ groups, respectively. A decrease in $\mathrm{Cp}_{2}$ (the heat capacity corresponding to local maximum at $T_{2}$ ) intensity is well visible in Fig. 3a for the representative from placebo group and a substantial changes in the temperature range $55-65^{\circ} \mathrm{C}$ in Fig. 3 b, which have appeared after the training combined with supplementation.

To illustrate and to evaluate the effect of a graded exercise to volitional fatigue and next the $1 \mathrm{~h}$ of rest, sets of three curves are shown in Fig. 4 for the $\mathrm{C}$ group before the training and in Fig. 5 for the $\mathrm{S}$ groups after the training
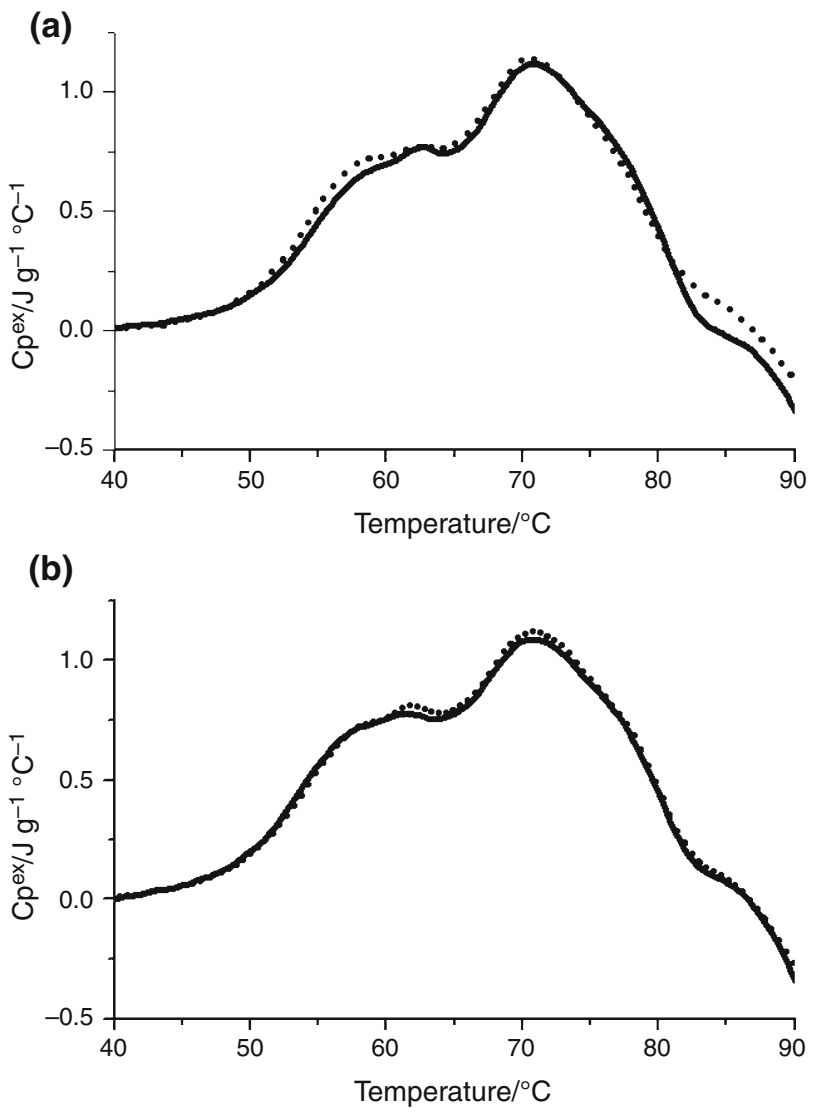

Fig. 2 a Comparison of mean serum DSC curves in pre-exercise stage before (solid lines) and after (dotted lines) the CrossFit training for the $\mathrm{C}$ group. $\mathbf{b}$ Comparison of mean serum DSC curves in preexercise stage before (solid lines) and after (dotted lines) the CrossFit training for the $\mathrm{S}$ group

and GTE supplementation. The comparison of these average curves indicates that the exercise performed by men slightly shifts the observed endothermic transition to lower temperatures. The effect visible in Fig. 4 has been 


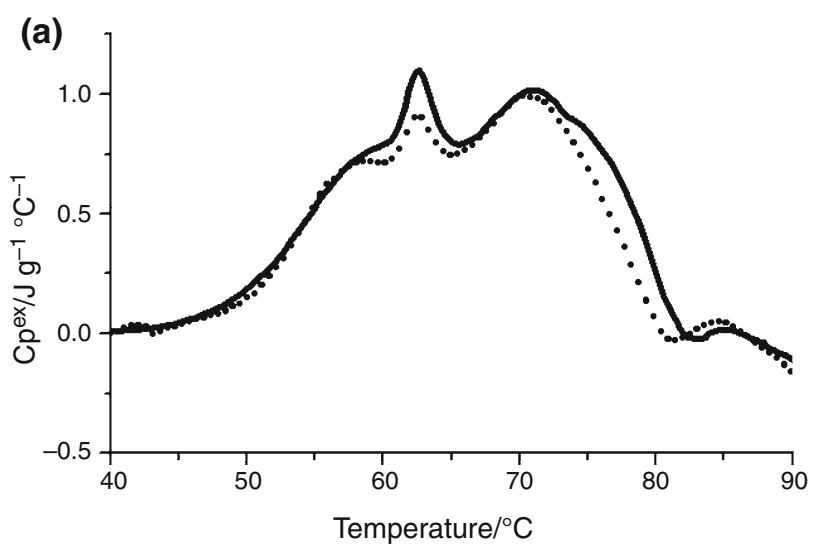

(b)

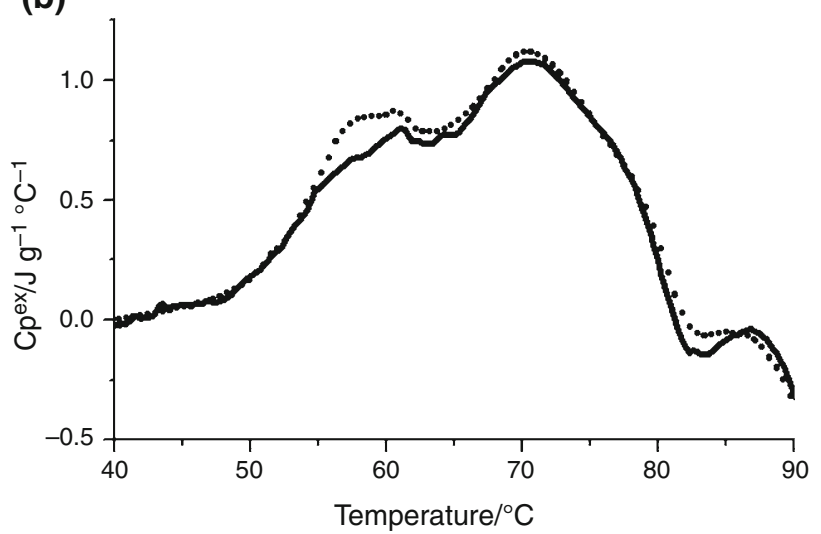

Fig. 3 a An example of substantial differences between serum DSC curves before (solid lines) and after (dotted lines) CF training for the representative from the $\mathrm{C}$ group. b An example of substantial differences between serum DSC curves before (solid lines) and after (dotted lines) CF training in combination with GTE supplementation

observed even weaker in the case of S group (not shown) and after the training in both groups. After the recovery period of $1 \mathrm{~h}$ changes recede, but for the $\mathrm{S}$ group in the second trial mean DSC curve for serum acquired after the rest does not practically differ from that corresponding to

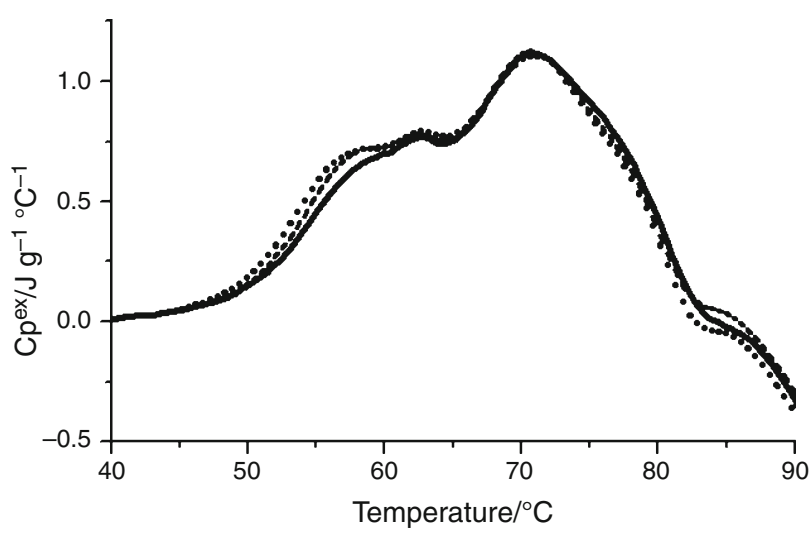

Fig. 4 Comparison of mean serum DSC curves in three stages: preexercise (solid lines), post-exercise (dotted lines) and after $1 \mathrm{~h}$ of rest (dashed dotted lines) before the CrossFit training for the $\mathrm{C}$ group

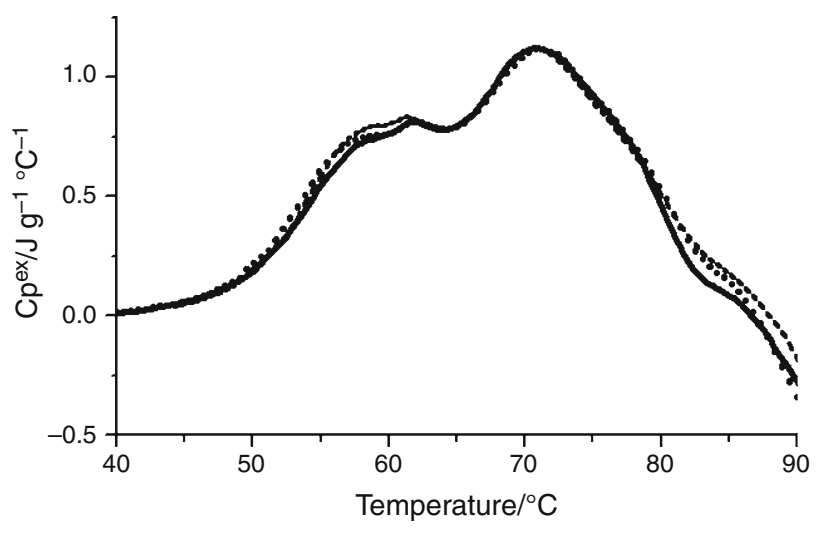

Fig. 5 Comparison of mean serum DSC curves in three stages: preexercise (solid lines), post-exercise (dotted lines) and after $1 \mathrm{~h}$ of rest (dashed dotted lines) after the CrossFit training for the $\mathrm{S}$ group

post-exercise serum (Fig. 5). It is worth mentioning that the response to exercise and rest varied substantially among individual participants of the study.

Modifications of serum DSC transition observed in current study due to the exercise and rest for well-trained participants are very small in contrast to relatively distinct changes found in our previous study for amateur cyclists [37]. The main reason for this poor response of serum proteins on strenuous exercise is probably a high physical performance of participants even before the training. CrossFit is extremely popular form of multimodal exercise training conducted at high intensity in order to improve endurance, strength and flexibility [40]. Data from available studies suggest that catechins can improve physical performance particularly in terms of endurance capacity and $\mathrm{VO}_{2 \mathrm{max}}$ in untrained subjects, but the same results cannot be reached in physically active people and welltrained athletes [5].

The mean values \pm SEM of parameters describing the endothermic denaturation transition for serum acquired from participants in the first (before the CrossFit training) and in the second (after the training, marked with additional letter $\mathrm{t}$ ) trial for $\mathrm{C}$ (control-placebo) and S (GTE supplemented) groups are presented in Figs. 6-9. The dependence of these parameters on the stage of experiment: before exercise (be), after exercise (ae) and after $1 \mathrm{~h}$ of rest (r1h), is illustrated.

Figure 6 suggests that the character of $T_{1}, T_{2}$ and $T_{3}$ dependences on the stage of experiment is very similar irrespective of the group and a completion of the training. No significant differences in $T_{\mathrm{m}}$ values have been observed between both groups in the adequate study periods. For both groups in both trials, post-exercise $T_{1}$ was somewhat lower, while post-exercise $T_{2}$ and $T_{3}$ were higher than before the exercise. Taking together results for both groups in both trials, the repeated measure ANOVA indicated 
Fig. 6 Mean values of $T_{1}, T_{2}$ and $T_{3}$ ( \pm s.e.) for different stages of experiment in both groups
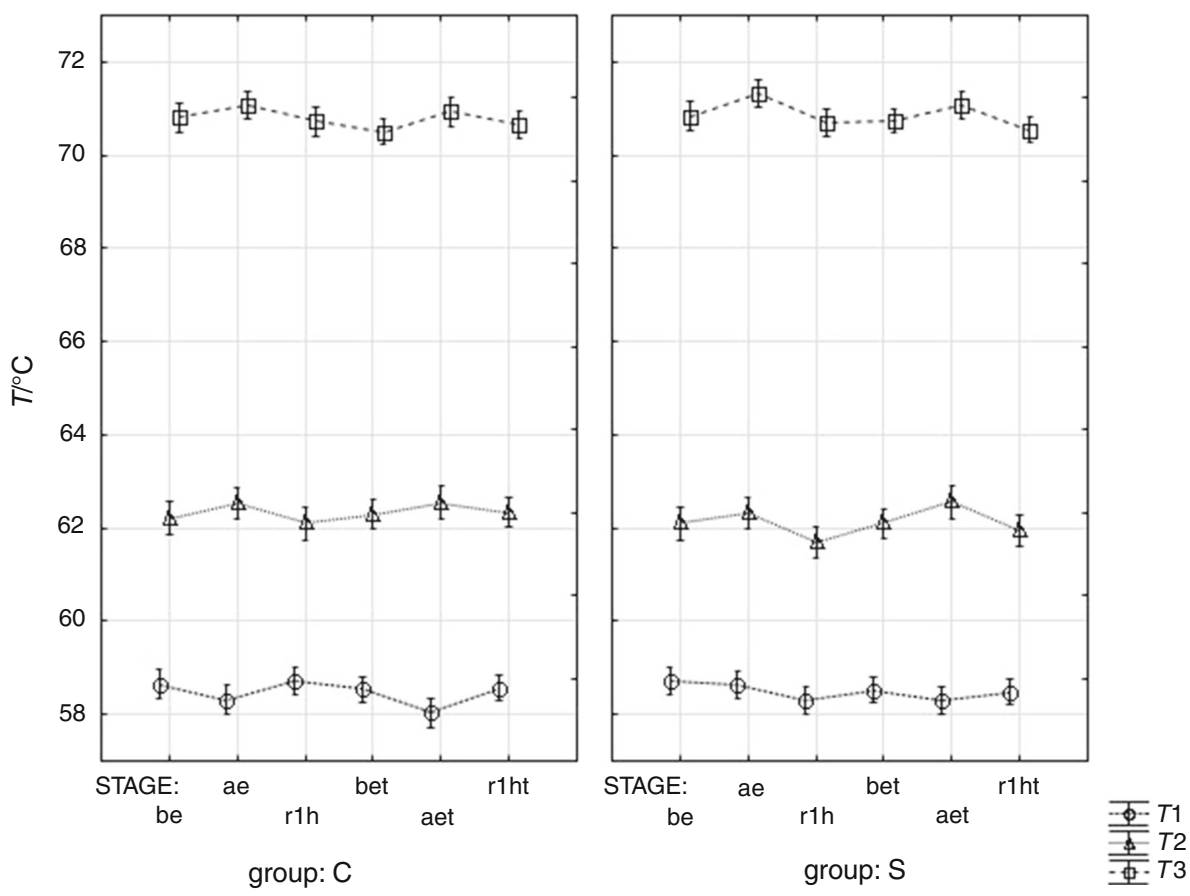

statistically significant differences between $T_{2}$ ( $p=0.00005)$ as well as between $T_{3}(p=0.00004)$ in different stages of experiment. According to post hoc Tukey's test, post-exercise values of $T_{2}$ and $T_{3}$ were significantly higher than after $1 \mathrm{~h}$ of rest. Considering additionally the factor "group," mentioned differences for $T_{2}$ were significant only in GTE-supplemented group. Moreover, post-exercise values of $T_{3}$ in both trials were significantly higher than before-exercise value in the second trial. Only for the $\mathrm{S}$ group post-exercise $T_{3}$ value in the first trial was significantly higher than values of $T_{3}$ obtained in both trials after the rest.

Changes in $C p_{1}, C p_{2}$ and $C p_{3}$ related to the stage of experiment, shown in Fig. 7, seem somewhat different for the $\mathrm{C}$ and $\mathrm{S}$ groups. However, suggested by Fig. 7 differences between both groups in $\mathrm{Cp}_{\mathrm{m}}$ mean values are not statistically significant. Interesting results have been found for $\mathrm{Cp}_{2}$ parameter. For the $\mathrm{S}$ group, after the training and supplementation $\mathrm{Cp}_{2}$ value is maintained at a constant, higher than in the first trial, level. Generally, taking the stage of experiment as a main factor, according to the repeated measure ANOVA, differences between mean $\mathrm{Cp}_{2}$ values are statistically significant $(p=0.047)$. Taking into consideration the data obtained for both groups, the preexercise $\mathrm{Cp}_{2}$ in the first trial has been found significantly lower than post-exercise value after the training $(p=0.02$ in post hoc Tukey's test).

The main origin of the local maximum $\left(T_{2}, \mathrm{Cp}_{2}\right)$ in the serum denaturation peak is probably haptoglobin, the protein from alpha-2 globulins fraction [25, 37]. Haptoglobin is an acute-phase protein, the synthesis of which is increased during inflammation. Haptoglobin binds free hemoglobin released from erythrocytes with high affinity and therefore inhibits its oxidative activity. In this study, significant negative correlations have been found between $\mathrm{VO}_{2 \max }$ after CF training and post-exercise $T_{2}(r=-0.52$; $p=0.03)$ as well as post-exercise $\mathrm{Cp}_{2} \quad(r=-0.68$; $p=0.03$ ) after the training. The first of said correlations was even higher in the control group $(r=-0.78)$, while the second in the supplemented group $(r=-0.86)$. Thus, higher $\mathrm{VO}_{2 \max }$ after the $\mathrm{CF}$ training implies a smaller increase in $T_{2}$ and $\mathrm{Cp}_{2}$ after the exercise.

The relationship between $\mathrm{VO}_{2 \max }$ and $\mathrm{Cp}_{2}$ intensity confirms the observation that a lack of $T_{2}$ local maximum has been most frequently accompanied by $\mathrm{VO}_{2 \max }$ higher than $50 \mathrm{~mL} \mathrm{~min}{ }^{-1} \mathrm{~kg}^{-1}$. Moreover, the case shown in Fig. 3a represents a man with the lowest value of $\mathrm{VO}_{2 \max }$ : 39 and $43 \mathrm{~mL} \mathrm{~min}^{-1} \mathrm{~kg}^{-1}$ before and after the $\mathrm{CF}$ training, respectively. Among all DSC curves recorded in the current study, it was the case with the highest $\mathrm{Cp}_{2}$ component. In accordance with the found negative correlation between $\mathrm{VO}_{2 \max }$ and $\mathrm{Cp}_{2}$, a decrease in $\mathrm{Cp}_{2}$ component has been observed due to the increase in $\mathrm{VO}_{2 \max }$ after the training.

Figures 8 and 9 indicate that trends of changes in the enthalpy of serum denaturation $(\Delta H)$ and HHW are very similar in both groups, but these values are bigger for the $\mathrm{S}$ group. An increase in $\Delta H$ and HHW after the exercise and a decrease after the recovery period have been observed in the first as well as in second trial for both groups. Statistically significant differences have been found only for HHW parameter due to "group" and "stage" factors 
Fig. 7 Mean values of $\mathrm{Cp}_{1}, \mathrm{Cp}_{2}$ and $\mathrm{Cp}_{3}( \pm$ s.e.) for different stages of experiment in both groups
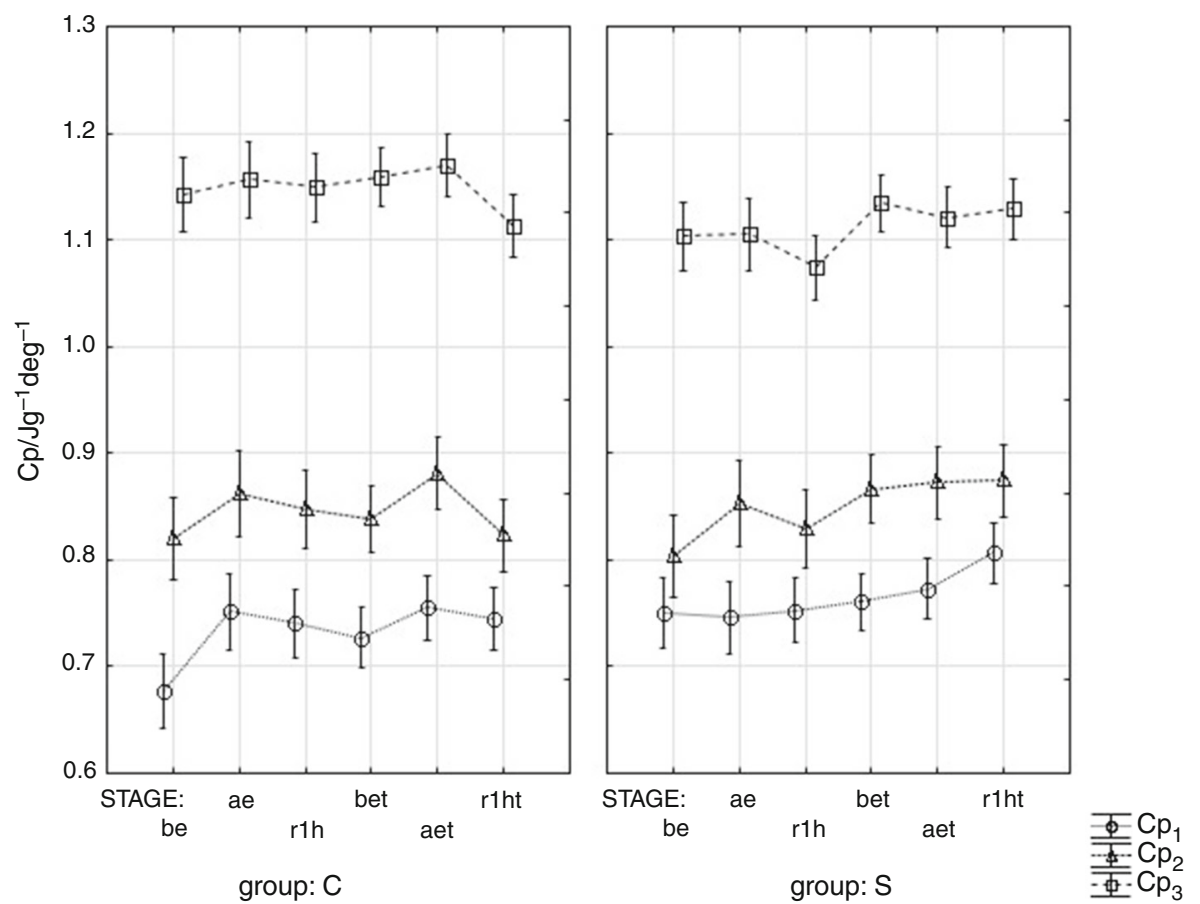

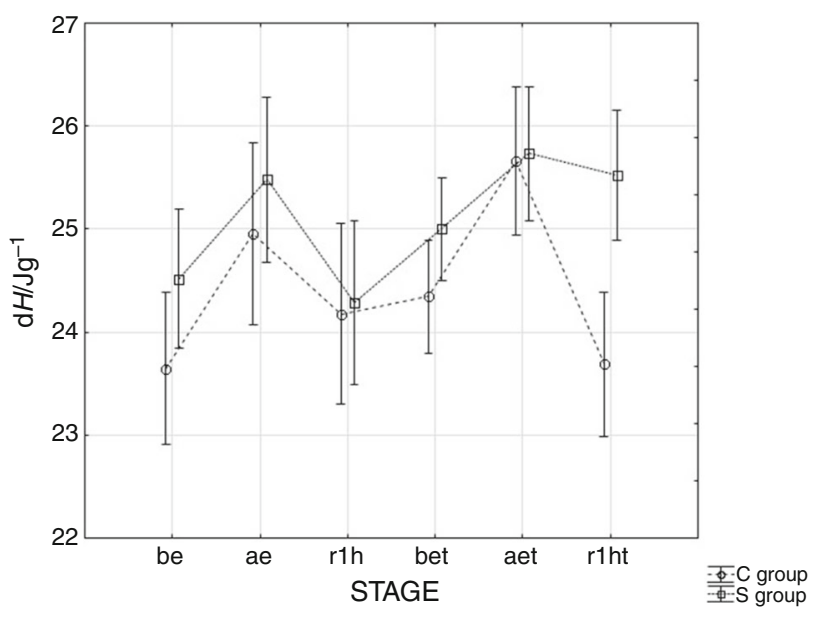

Fig. 8 Mean values of $\Delta H$ ( \pm s.e.) for different stages of experiment in both groups

( $p=0.004$ and $p=0.01$, respectively). Additionally, post hoc Tukey's test has revealed significantly broader serum denaturation transition in the post-exercise stage in the second trial for the $\mathrm{S}$ group than in all stages for the $\mathrm{C}$ group except these after the exercise.

An explanation of why obtained HHW values are higher for the $\mathrm{S}$ than for the $\mathrm{C}$ group and after the exercise is not simple, and we are not able to clarify this problem at present. Generally, smaller HHW indicates greater cooperativity of the observed thermal transition, but exothermic proteins aggregation following their endothermic unfolding may also cause a narrowing of the denaturation transition. Another reason of differences in the width of peak

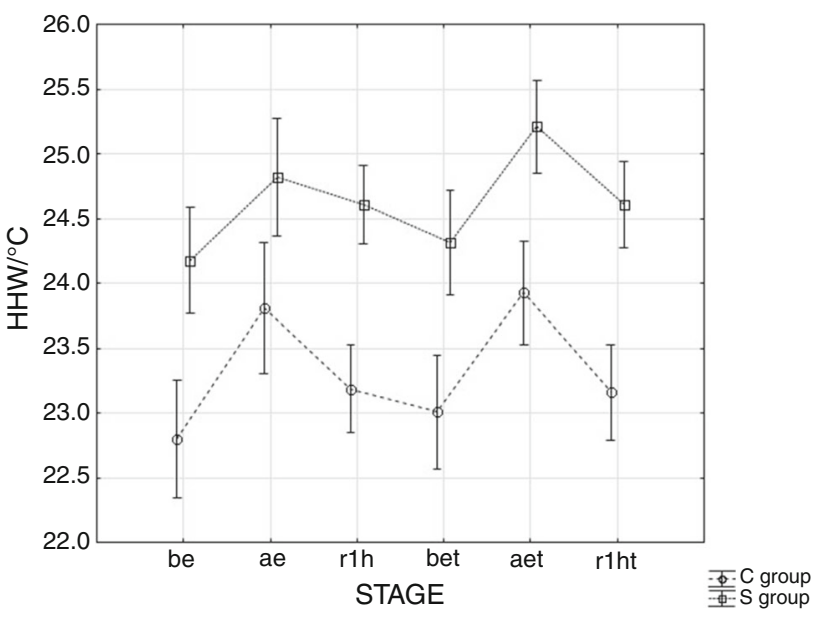

Fig. 9 Mean values of HHW ( \pm s.e.) for different stages of experiment in both groups

represented serum denaturation may be the proportion of constituent proteins or modification of their structures, for example, due to oxidation. Slightly higher values of $\mathrm{Cp}_{3}$ for the $\mathrm{C}$ than for the $\mathrm{S}$ group (except the after recovery stage in the second trial) suggest a higher contribution from gamma globulins in the control group. However, more prominent cause of considered broadening of serum transition seems to be connected with its low temperature range (see Figs. 1, 4), where the main contribution comes from albumin, the most abundant human serum protein. An increase in albumin level after the exercise was found in our earlier study [37]. Additionally, conformational changes in albumin molecule due to the binding of fatty acids or 
other ligands as well as protein glycation affect its folding and stability [41, 42]. This heterogeneity of the albumin molecule may cause the extension of thermal unfolding range. Albumin plays, among others, key antioxidant functions. In normal conditions, oxidation of albumin involves only a minor part of the protein. However, due to oxidative stress more oxidants react with albumin and give rise to oxidized albumin level. Reported by Musante et al. [43], value of $\Delta H$ for oxidized albumin was bigger and thermal melting peak was shifted significantly to the higher temperature range $\left(T_{\mathrm{m}}\right.$ above $80{ }^{\circ} \mathrm{C}$ was observed) in comparison with normal albumin. The oxidative stress caused by strenuous physical exercise may lead to the increased fraction of oxidized albumin in serum [44]. This may be one of the reasons for broadening the temperature range of serum denaturation because of the additional contribution from this form of albumin also to the higher temperature side of serum endothermic transition.

\section{Conclusions}

It is now generally accepted that strenuous physical exercise can induce oxidative stress in humans. Antioxidants supplementation may support endogenous defense system and prevent or reduce oxidative stress, decrease muscle damage and improve exercise performance. The results of the present study support this view to a certain extent. According to the obtained data, GTE supplementation parallel with a strenuous $\mathrm{CF}$ training prevents the posttraining increase in creatine kinase (CK) activity observed for the placebo group. Thus, GTE can mitigate the muscle damage induced by physical exercise.

DSC results do not indicate the clear effects of training or GTE supplementation on serum denaturation transition. However, thermodynamic parameters of this thermal transition change in a characteristic way during the performed experiment. While comparing these parameters for the $\mathrm{C}$ and $\mathrm{S}$ groups in two trials: before and after the $\mathrm{CF}$ training and in three phases: pre-exercise, post-exercise and after $1 \mathrm{~h}$ of resting recovery, some significant differences have been found. In particular, in both trials, taken together for both groups post-exercise values of $T_{2}$ as well as $T_{3}$ have appeared significantly higher than corresponding values after the recovery. The post-exercise values of $T_{3}$ in both trials have also been stated as significantly higher than before-exercise $T_{3}$ value in the second trial. No significant differences in $T_{\mathrm{m}}$ values have been observed between both groups in corresponding study periods. Taking into account the data obtained for both groups, the pre-exercise $\mathrm{Cp}_{2}$ before the training has been shown significantly lower than post-exercise $\mathrm{Cp}_{2}$ after the training. Comparing the width of the serum denaturation transition, it has been higher for the $\mathrm{S}$ than for the $\mathrm{C}$ group in all stages of the experiment. The broadest transition (the highest mean HHW value) has been observed after the exercise in the second trial for the $\mathrm{S}$ group. It is not clear why the exercise in conjunction with CF training and GTE supplementation leads to a reduction in the transition cooperativity.

A summary of the biochemical and calorimetric data has revealed the relationship between $\mathrm{VO}_{2 \max }$ and $\mathrm{Cp}_{2}$ intensity. The findings of statistical analysis indicate significant negative correlation between these variables. The overall data presented in this study suggest that in terms of $\mathrm{VO}_{2 \max }$ catechins do not improve physical performance in physically active, well-trained men. However, further studies are needed to clarify this problem.

Open Access This article is distributed under the terms of the Creative Commons Attribution 4.0 International License (http://crea tivecommons.org/licenses/by/4.0/), which permits unrestricted use, distribution, and reproduction in any medium, provided you give appropriate credit to the original author(s) and the source, provide a link to the Creative Commons license, and indicate if changes were made.

\section{References}

1. Peternelj TT, Coombes JS. Antioxidant supplementation during exercise training: beneficial or detrimental? Sports Med. 2011;41:1043-69.

2. Gomez-Cabrera MC, Ristov M, Viña J. Antioxidant supplements in exercise: worse than useless? Am J Physiol Endocrinol Metab. 2012;302:E476-7.

3. Brisswalter J, Louis J. Vitamin supplementation benefits in master athletes. Sports Med. 2014;44(3):311-8.

4. Pingitore A, Lima GPP, Mastorci F, Quinones A, Iervasi G, Vassalle C. Exercise and oxidative stress: potential effects of antioxidant dietary strategies in sports. Nutrition. 2015;31:916-22.

5. Malaguti M, Angeloni C, Hrelia S. Polyphenols in exercise performance and prevention of exercise-induced muscle damage. Oxid Med Cell Longev. Hindawi Publishing Corporation. 2013, Article ID 825928. doi: 10.1155/2013/825928.

6. Myburgh KH. Polyphenol supplementation: benefits for exercise performance or oxidative stress? Sports Med. 2014;44(Suppl 1):S57-70.

7. Yavari A, Javadi M, Mirmiran P, Bahadoran Z. Exercise-induced oxidative stress and dietary antioxidants. Asian J Sports Med. 2015;6(1):1-7.

8. Rahal A, Kumar A, Singh V, Yadav B, Tiwari R, Chakraborty S, et al. Oxidative stress, prooxidants, and antioxidants: the interplay. Biomed Res Int 2014;2014, Article ID 761264. doi:10.1155/ 2014/761264.

9. Steinbacher P, Eckl P. Impact of oxidative stress on exercising skeletal muscle. Biomolecules. 2015;5:356-77.

10. Banfi G, Colombini A, Lombardi G, Lubkowska A. Metabolic markers in sports medicine. Adv Clin Chem. 2012;56(1):24.

11. Yang CS, Landau JM. Effects of tea consumption on nutrition and health. J Nutr. 2000;130:2409-12.

12. Sae-Tan S, Grove KA, Lambert JD. Weight control and prevention of metabolic syndrome by green tea. Pharmacol Res. 2011;64(2):146-54. 
13. Park HJ, DiNatale DA, Chung MY, Park YK, Lee JY, Koo SI, O'Connor M, Manautou JE, Bruno RS. Green tea extract attenuates hepatic steatosis by decreasing adipose lipogenesis and enhancing hepatic antioxidant defenses in ob/ob mice. J Nutr Biochem. 2011;22(4):393-400.

14. Singh BN, Shankar S, Srivastava RK. Green tea catechin, epigallocatechin-3-gallate (EGCG): mechanisms, perspectives and clinical applications. Biochem Pharmacol. 2011;82(12):1807-21.

15. Zhang Y, Zhang D, Zhu B, Zhang H, Sun Y, Sun C. Effects of dietary green tea polyphenol supplementation on the health of workers exposed to high-voltage power lines. Environ Toxicol Pharmacol. 2016;46:183-7.

16. Panza VS, Wazlawik E, RicardoSchütz G, Comin L, Hecht KC, da Silva EL. Consumption of green tea favorably affects oxidative stress markers in weight-trained men. Nutrition. 2008;24(5):433-42.

17. Richards JC, Lonac MC, Johnson TK, Schweder MM, Bell C. Epigallocatechin-3-gallate increases maximal oxygen uptake in adult humans. Med Sci Sports Exerc. 2010;42(4):739-44.

18. Jówko E, Sacharuk J, Balasinska B, et al. Green tea extract supplementation gives protection against exercise-induced oxidative damage in healthy men. Nutr Res. 2011;31(11):813-21.

19. Jówko E, Sacharuk J, Balasinska B, et al. Effect of a single dose of green tea polyphenols on the blood markers of exercise-induced oxidative stress in soccer players. Int J Sport Nutr Exerc Metab. 2012;22(6):486-96.

20. Sugita M, Kapoor MP, Nishimura A, Okubo T. Influence of green tea catechins on oxidative stress metabolites at rest and during exercise in healthy humans. Nutrition. 2016;32:321-31.

21. Herrliger KA, Chirouzes DM, Ceddia MA. Supplementation with a polyphenolic blend improves post-exercise strength recovery and muscle soreness. Food Nutr Res. 2015;59:1-10.

22. Zapora E, Holub M, Waszkiewicz E, Dabrowska M, Skrzydlewska E. Green tea effect on antioxidant status of erythrocytes and on haematological parameters in rats. Bull Vet Inst Pulawy. 2009;53(1):139-45.

23. Arent SM, Senso M, Golem DL, McKeever KH. The effects of the aflavin-enriched black tea extract on muscle soreness, oxidative stress, inflammation, and endocrine responses to acute anaerobic interval training: a randomized, double-blind, crossover study. J Int Soc Sports Nutr. 2010;7(1):11.

24. Hodgson AB, Randell RK, Boon N, et al. Metabolic response to green tea extract during rest and moderate-intensity exercise. J Nutr Biochem. 2013;24(1):325-34.

25. Garbett NC, Miller JJ, Jenson AB, Chaires JB. Calorimetry outside the box: a new window into the plasma proteome. Biophys J. 2008;94:1377-83.

26. Garbett NC, Mekmaysy CS, Helm W, Jenson AB, Chaires JB. Differential scanning calorimetry of blood plasma for clinical diagnosis and monitoring. Exp Mol Pathol. 2009;86:186-91.

27. Michnik A, Drzazga Z, Michalik K, Barczyk A, Santura I, Sozańska E, Pierzchała W. Differential scanning calorimetry study of blood serum in chronic obstructive pulmonary disease. J Therm Anal Calorim. 2010;102:57-60.

28. Lőrinczy D, editor. Thermal analysis in medical application. Budapest: Akadémiai Kiadó; 2011.
29. Todinova S, Krumova S, Gartcheva L, Robeerts $\mathrm{Ch}$, Taneva SG. Microcalorimetry of blood serum proteome: a modified interaction network in the multiple myeloma case. Anal Chem. 2011;83:7992-8.

30. Todinova S, Krumova S, Kurtev P, Dimitrov V, Djongov L, Dudunkov Z, Taneva SG. Calorimetry-based profiling of blood plasma from colorectal cancer patients. Biochim Biophys Acta. 2012;1820:1879-85.

31. Fekecs T, Zapf I, Ferencz A, Lőrinczy D. Differential scanning calorimetry (DSC) analysis of human plasma in melanoma patients with or without regional lymph node metastases. J Therm Anal Calorim. 2012;108:149-52.

32. Krumova S, Rukova B, Todinova S, Gartcheva L, Milanova V, Toncheva D, Taneva SG. Calorimetric monitoring of the serum proteome in schizophrenia patients. Thermochim Acta. 2013;572:59-64.

33. Moezzi M, Fekecs T, Zpf I, Ferencz A, Lőrinczy D. Differential scanning calorimetry (DSC) analysis of human plasma in different psoriasis stages. J Therm Anal Calorim. 2013;111:1801-4.

34. Garbett NC, Mekmaysy CS, DeLeeuw L, Chaires JB. Clinical application of plasma thermograms. Utility, practical approaches and considerations. Methods. 2015;76:41-50.

35. Vega S, Garcia-Gonzalez MA, Lanas A, Velazquez-Campoy A, Abian O. Deconvolution analysis for classifying gastric adenocarcinoma patients based on differential scanning calorimetry serum thermograms. Sci Rep. 2015;5:7988. doi:10.1038/srep07988.

36. Ferencz A, Lörinczy D. DSC measurements of blood plasma on patients with chronic pancreatitis and operable and inoperable pancreatic adenocarcinoma. J Therm Anal Calorim. 2017;. doi:10.1007/s10973-016-5371-4.

37. Michnik A, Drzazga Z, Poprzęcki S, Czuba M, Kempa K, Sadowska-Krępa E. DSC serum profiles of sportsmen. J Therm Anal Calorim. 2013;113:365-70.

38. Michnik A, Drzazga Z, Sadowska-Krępa E, Kłapcińska B. Calorimetric monitoring of the effect of endurance training and testosterone treatment on rat serum denaturation transition. J Therm Anal Calorim. 2014;115:2231-7.

39. Michnik A, Drzazga Z. Thermal denaturation of mixtures of human serum proteins DSC study. J Therm Anal Calorim. 2010;101:513-8.

40. Glassman G. The CrossFit training guide. CrossFit J. 2010;9:1-115.

41. Michnik A, Michalik K, Kluczewska A, Drzazga Z. Comparative DSC study of human and bovine serum albumin. J Therm Anal Calorim. 2006;84:113-7.

42. Vetter SW, Indurthi VSK. Moderate glycation of serum albumin affects folding, stability, and ligand binding. Cin Chim Acta. 2011;412:2105-16.

43. Musante L, Bruschi M, Candiano G, Petretto A, Dimasi N, Del Boccio P, Urbani A, Rialdi G, Ghiggeri GM. Characterization of oxidation end product of plasma albumin 'in vivo'. Biochem Biophys Res Commun. 2006;349(2):668-73.

44. Imai H, Hayashi T, Negawa T, Nakamura K, Tomida M, Koda K, et al. Strenuous exercise-induced change in redox state of human serum albumin during intensive kendo training. Jpn J Physiol. 2002;52:135-40. 